\title{
Anisotropic flow measurements from RHIC to SIS
}

\author{
Arkadiy Taranenko ${ }^{1, *}$ \\ ${ }^{1}$ National Research Nuclear University MEPhI, Moscow, Russia
}

\begin{abstract}
Relativistic heavy-ion collisions provide a unique opportunity to study the expansion dynamics and the transport properties of the produced strongly interacting matter. This article reviews the recent results of anisotropic flow measurements for collision energies from $\sqrt{s_{\mathrm{NN}}}=200$ to $2 \mathrm{GeV}$.
\end{abstract}

\section{Introduction}

The heavy-ion experiments at the Relativistic Heavy Ion Collider (RHIC) and the Large Hadron Collider (LHC) have established the existence of a strongly coupled Quark Gluon Plasma (sQGP) [1,2], a new state of nuclear matter with partonic degrees of freedom and with low specific shear viscosity $\eta / s$, i.e. the ratio of shear viscosity $\eta$ to entropy density $[3,4]$. Lattice QCD calculations [5] indicate that the quark-hadron transition is a smooth crossover at top RHIC energies and above (small $\mu_{B}$ ). The possible region of a first order phase transition is expected at larger values of $\mu_{B}$ (lower beam energies), suggesting the existence of a critical end point (CEP) [6]. Thus, a current strategy for experimental mapping of the QCD phase diagram is centered on beam energy scans (BES), which sample reaction trajectories with the broadest possible range of $\mu_{B}$ and $\mathrm{T}$ values. The existing BES programs include: programs BES-I and BES-II of STAR experiment at RHIC for Au+Au collisions at $\sqrt{s_{\mathrm{NN}}}=3-200$ GeV (collider + fixed target) [7-9] and program of NA61/SHINE fixed target experiment at SPS for the different colliding systems $(\mathrm{Be}+\mathrm{Be}, \mathrm{Ar}+\mathrm{Sc} \mathrm{Xe}+\mathrm{La}, \mathrm{Pb}+\mathrm{Pb})$ at $\sqrt{s_{\mathrm{NN}}}=5.1-17.3$ $\mathrm{GeV}$ [10]. The future experiments at Nuclotron-based Ion Collider fAciilty (NICA) [11] and Facility for Antiproton and Ion Research (FAIR) [12] will explore the phase diagram at high baryon density region.

The anisotropic flow, as manifested by the anisotropic emission of particles in the plane transverse to the beam direction, is one of the important observable sensitive to the transport properties of the strongly interacting matter: the equation of state (EOS), the speed of sound $\left(c_{S}\right)$ and the value of $\eta / s[3,4]$. The azimuthal anisotropy of produced particles can be quantified by the Fourier coefficients $v_{n}$ in the expansion of the particles azimuthal distribution as: $d N / d \phi \propto 1+\sum_{n=1} 2 v_{n} \cos \left(n\left(\phi-\Psi_{n}\right)\right)$ [13-15], where $n$ is the order of the harmonic, $\phi$ is the azimuthal angle of particles of a given type, and $\Psi_{n}$ is the azimuthal angle of the $n$ th-order event plane. The $n^{\text {th }}$-order flow coefficients $v_{n}$ can be calculated as $v_{n}=\left\langle\cos \left[n\left(\varphi-\Psi_{n}\right)\right]\right\rangle$, where the brackets denote an average over particles and events. In this work, we briefly review and discuss the recent results of the measurements of directed $\left(v_{1}\right)$, elliptic $\left(v_{2}\right)$ and triangular $\left(v_{3}\right)$ flow for collision energies from $\sqrt{s_{\mathrm{NN}}}=200 \mathrm{GeV}$ (RHIC) to $2 \mathrm{GeV}$ (SIS). Elliptic $\left(v_{2}\right)$ and triangular $\left(v_{3}\right)$ are the dominant flow signals and have been studied very

\footnotetext{
*e-mail: AVTaranenko@mephi.ru
} 
extensively both at top RHIC and LHC energies. Relativistic viscous hydrodynamics has been successful in describing the observed $v_{2}$ and $v_{3}$ signals for produced particles in the collisions of heavy-ion systems and the overall good agreement between data and model calculations can be reached for small values of $\eta / s$ closed to the lower conjectured bound of $1 / 4 \pi[3,4,16]$. In this model framework, the values of the coefficients $v_{n}$ (for $p_{T}<3 \mathrm{GeV} / \mathrm{c}$ ) have been attributed to an eccentricity-driven hydrodynamic expansion of the plasma produced in the collision zone. That is, a finite eccentricity moment $\varepsilon_{n}$ drives uneven pressure gradients in- and out of the event plane $\psi_{n}$, and the resulting expansion leads to the anisotropic flow of particles about this plane. The event-by-event geometric fluctuations in its initial density distribution are found to be responsible for finite elliptic flow signal $v_{2}$ in the collisions with almost zero impact parameter, and the presence of odd harmonic moments in the initial geometry $\varepsilon_{n}$ and final momentum anisotropy $v_{n}[14,15]$. The proportionality constant between $v_{n}$ and $\varepsilon_{n}$ is found to be sensitive to the transport properties of the matter such as as the equation of state and the specific shear viscosity $\eta / s$. The shear viscosity suppresses higher order harmonic flow coefficients $v_{n>2}$ more strongly than the elliptic flow signal $v_{2}$. The $v_{n}$ data at top RHIC energy and LHC seem to follow the "acoustic scaling" for anisotropic flow, which suggests that viscous corrections to $v_{n} / \varepsilon_{n}$ grow exponentially as $n^{2}$ and $1 /(\bar{R} T)$ $[17,18,24]$ :

$$
\frac{v_{n}\left(p_{T}, \text { cent }\right)}{\varepsilon_{n}(\text { cent })} \propto \exp \left[-n^{2} \beta^{\prime}\right], \beta^{\prime} \propto \frac{\eta}{s} \frac{1}{\bar{R} T} \propto \frac{\eta}{s} \frac{1}{\left(d N_{c h} / d \eta\right)^{1 / 3}}
$$

where $\mathrm{T}$ is the temperature, $\bar{R}$ is the transverse size of the collision zone. For a given harmonic number $\mathrm{n}$, this equation indicates a characteristic linear dependence of $\ln \left(v_{n} / \varepsilon_{n}\right)$ on $1 /(\bar{R} T)$, with a slope proportional to $\eta / s[17,18,24]$. The dimensionless size $\bar{R} T$ is approximately proportional to $\left(d N_{c h} / d \eta\right)^{1 / 3}$, where $d N_{c h} / d \eta$ is the charge particle multiplicity density [25]. The recent data from STAR collaboration indicate that $v_{2} / \varepsilon_{2}$ versus $\left(N_{c h}\right)^{-1 / 3}$ has the same slope for charged hadrons from six different colliding systems: $\mathrm{U}+\mathrm{U}, \mathrm{Au}+\mathrm{Au}$, $\mathrm{Cu}+\mathrm{Au}, \mathrm{Cu}+\mathrm{Cu}, \mathrm{d}+\mathrm{Au}$ and $\mathrm{p}+\mathrm{Au}$ collisions at top RHIC energy [20], see left panel of Fig. 1. According to the "acoustic scaling" the value of this slope is proportional to $\eta / s$. The validation of the scaling relations for so many different colliding systems strongly support the hydrodynamic picture and may indicate that the value of $\eta / s$ is very similar for different systems.
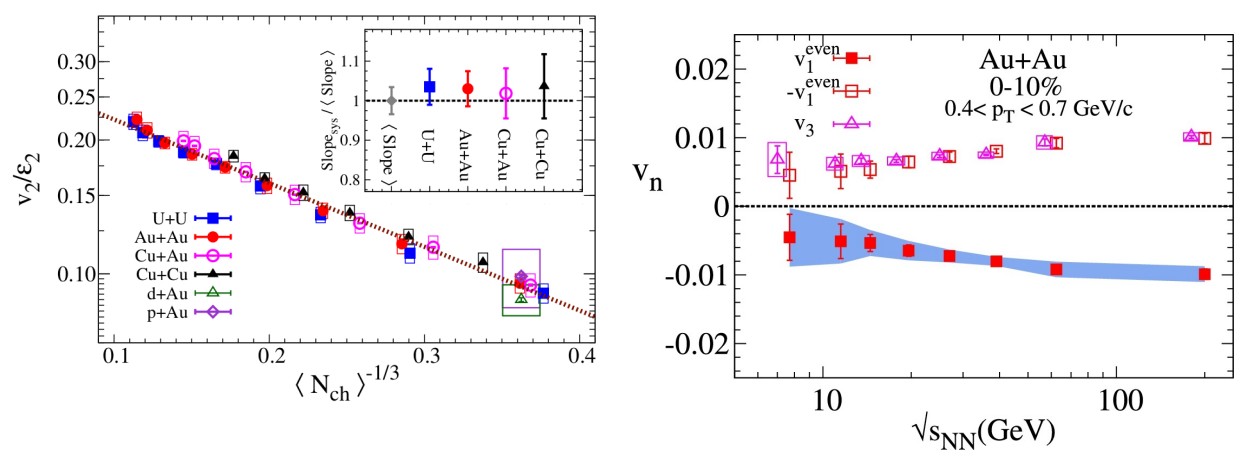

Figure 1. Left: $v_{2} / \varepsilon_{2}$ versus $\left(N_{c h}\right)^{-1 / 3}$ for charged hadrons from $\mathrm{U}+\mathrm{U}, \mathrm{Au}+\mathrm{Au}, \mathrm{Cu}+\mathrm{Au}, \mathrm{Cu}+\mathrm{Cu}, \mathrm{d}+\mathrm{Au}$ and $\mathrm{p}+\mathrm{Au}$ collisions at top RHIC energy. The picture with STAR data was taken from [20]. The dotted line represents an exponential fit to the data with Eq.1. Right: STAR data for $\sqrt{s_{\mathrm{NN}}}$ dependence of $v_{1}^{\text {even }}$ and $v_{3}$ for charged hadrons with $0.4<p_{T}<0.7 \mathrm{GeV} / \mathrm{c}$ in $0-10 \%$ central $\mathrm{Au}+\mathrm{Au}$ collisions [19] 
There are two components in directed flow $v_{1}(y)=v_{1}^{\text {odd }}(y)+v_{1}^{\text {even }}(y)[19,22]$. Rapidity-odd component of directed flow $v_{1}^{\text {odd }}$ is the first harmonic coefficient in the Fourier expansion of the final-state azimuthal distribution of particles relative to the reaction plane (a plane defined by the impact parameter vector and the beam direction) and describes a collective sideward motion of emitted particles $v_{1}^{o d d}(y)=-v_{1}^{\text {odd }}(-y)$. In symmetric collisions, such as $\mathrm{Au}+\mathrm{Au}$, the rapidity-even component $v_{1}^{\text {even }}\left(v_{1}^{\text {even }}(y)=v_{1}^{\text {even }}(-y)\right)$ arises from event-by-event fluctuations in the initial nuclei and it is proportional to the fluctuations-driven dipole asymmetry $\varepsilon_{1}$ of the system [21]. Similar to $v_{2}$ and $v_{3}$ the magnitude of $v_{1}^{\text {even }}$ is sensitive to $\eta / s$. The excitation functions for $v_{1}^{\text {even }}$ and $v_{3}$ of charged hadrons from $0-10 \%$ central $\mathrm{Au}+\mathrm{Au}$ collisions are shown in the right panel of Fig. 1. The results were obtained by STAR experiment during the BES-I scan at RHIC [19]. The $v_{1}^{\text {even }}$ data were reflected about zero to facilitate a comparison of the magnitudes. The comparison indicates very similar magnitudes and $\sqrt{s_{\mathrm{NN}}}$ dependence for $\left|v_{1}^{\text {even }}\right|$ and $v_{3}$. Both signals decrease with decreasing $\sqrt{s_{\mathrm{NN}}}$. The excitation functions of $v_{1}^{\text {even }}$ and $v_{3}$ are expected to provide new experimental input to ongoing theoretical efforts to discern between different initial-state models and make precision extractions of $\eta / s(T)$ [19].

\section{Directed flow}

The search for the predicted first-order phase transition between hadronic and QGP phases is one of the goals of the present beam energy scan programs at RHIC and SPS [7, 8]. Such a transition can also be characterized by a dramatic drop in the pressure, or a softening of the Equation of State (EOS) [32]. The signals like anisotropic flow are very promising due to their sensitivity to EOS. The rapidity-odd component of directed flow ( $v_{1}^{\text {odd }}$ ) can probe the very early stages of the collision as it is generated during the passage time of the two colliding nuclei $t_{\text {pass }}=2 R /\left(\gamma_{s} \beta_{s}\right)$, where $R$ is the radius of the nucleus at rest, $\beta_{s}$ is the spectator velocity in c.m. and $\gamma_{s}$ the corresponding Lorentz factor, respectively. Both hydrodynamic and transport model calculations indicate that the directed flow of charged particles, especially baryons at midrapidity, is very sensitive to the equation of state [31,32]. The slope of the rapidity dependence $d v_{1} / d y$ close to mid-rapidity is a convenient way to characterize the overall magnitude of the rapidity-odd component of directed flow signal [22]. A minimum in $d v_{1} / d y$ in the midrapidity region $(y \sim 0)$ could be related to the softening of equation-of-state due to the first order phase transition between hadronic matter and QGP [7, 8, 22, 31, 32]. The recent directed flow results from the STAR BES-I program at RHIC $\left(\sqrt{s_{\mathrm{NN}}}=7.7-200\right.$ $\mathrm{GeV}$ ) seem to support this prediction, both protons and $\Lambda$ hyperons $d v_{1} / d y$ show a minimum around $\sqrt{s_{\mathrm{NN}}}=10-20 \mathrm{GeV}$ [28], see left panel of Fig. 2. In general, the assumption of purely hadronic physics is disfavored by the comparison of experimental $d v_{1} / d y$ results and predictions from current state-of-the-art models [7, 22, 28]. However, all current models are not able to reproduce the basic trends of $\sqrt{s_{\mathrm{NN}}}$ dependence of baryon $d v_{1} / d y$-slope reported by STAR experiment. Thus, further progress in the area of model calculations of $v_{1}(y)$ is needed. On the experimental side, one need to perform high-statistics differential measurements of $v_{1}$ as a function of centrality, $p_{T}$, rapidity for different particle species. The statistics achieved in RHIC BES-I allow to focus the analysis $d v_{1} / d y$ only on a single wide centrality bin $10-40 \%$, see right panel of Fig. 2. The upper green curve in this panel shows the approximate upper bound of the measured $d v_{1} / d y$ range for protons in central collisions (0-10\%), and the lower green curve shows the approximate lower bound for peripheral collisions (60-80\%). These bounds based on the preliminary STAR results [37, 42] and they indicate a remarkably strong centrality dependence. Moreover, for low energies the $d v_{1} / d y$ slope for protons changes sign as a function of centrality. All these high-statistics measurements of $v_{1}$ will possible when STAR BES-II data become available. 

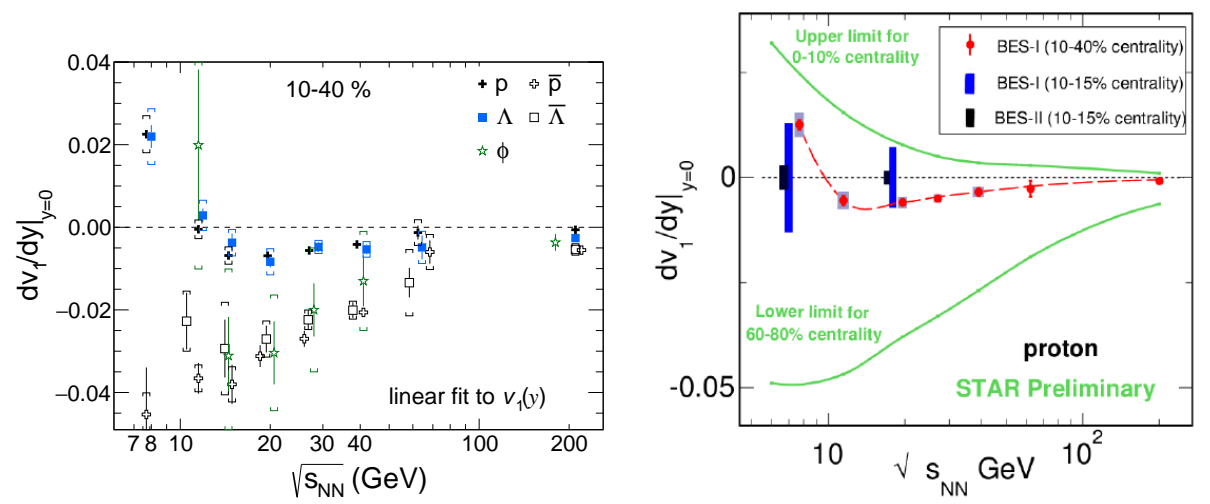

Figure 2. Left: STAR results for the beam energy dependence of the slope of rapidity-odd $d v_{1} / d y$ near mid-rapidity for protons, anti-protons, $\Lambda, \bar{\Lambda}$ and $\phi$ mesons from mid-central (10-40\%) Au+Au collisions. Figure is taken from [28]. Right: Expected centrality dependence for proton $d v_{1} / d y$ near midrapidity for $\mathrm{Au}+\mathrm{Au}$ collisions (green lines) based on STAR preliminary data from BES-I program. Figure is taken from [42]

The program of NA61/SHINE fixed target experiment at SPS(CERN) allows to perform the beam energy scan for different colliding systems $(\mathrm{Be}+\mathrm{Be}, \mathrm{Ar}+\mathrm{Sc} \mathrm{Xe}+\mathrm{La}, \mathrm{Pb}+\mathrm{Pb})$ in the energy range of $13-150 \mathrm{~A} \mathrm{GeV} / \mathrm{c}\left(\sqrt{s_{\mathrm{NN}}}=5.1-17.3 \mathrm{GeV}\right)$. This also allows one to extend the flow measurements carried out by STAR RHIC beam energy scan (BES) program to a wide rapidity range, extending up to the forward region where projectile nucleon spectators appear. Fig. 3(left) shows the preliminary data of NA61/SHINE collaboration for the centrality dependence of $d v_{1} / d y$ near midrapidity for protons and charged pions from $\mathrm{Pb}+\mathrm{Pb}$ collisions at $30 \mathrm{AGeV}\left(\sqrt{s_{\mathrm{NN}}}=7.65 \mathrm{GeV}\right)$. The data show that centrality dependence of $d v_{1} / d y$ is very strong and $d v_{1} / d y$ for protons is changing sign as a function of centrality. The right panel of Fig. 3 shows the $p_{T}$ dependence of $v_{1}$ of protons and charged pions $\left(\pi^{+}\right.$and $\left.\pi^{-}\right)$from $15-35 \%$ central $\mathrm{Pb}+\mathrm{Pb}$ collisions at $30 \mathrm{AGeV}[10]$.
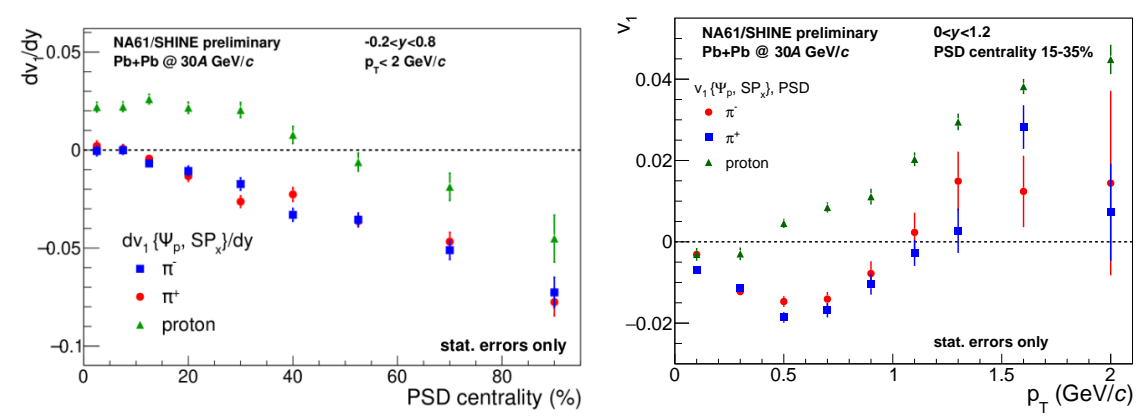

Figure 3. Left: Centrality dependence for $d v_{1} / d y$ near midrapidity for protons and charged pions from $\mathrm{Pb}+\mathrm{Pb}$ collisions at $30 \mathrm{AGeV}\left(\sqrt{s_{\mathrm{NN}}}=7.65 \mathrm{GeV}\right)$. Right: $p_{T}$ dependence of $v_{1}$ of protons and charged pions $\left(\pi^{+}\right.$and $\left.\pi^{-}\right)$from $15-35 \%$ central $\mathrm{Pb}+\mathrm{Pb}$ collisions at $30 \mathrm{AGeV}\left(\sqrt{s_{\mathrm{NN}}}=7.65 \mathrm{GeV}\right)$. [10] 


\section{Elliptic flow}

The elliptic flow $v_{2}$ is one of the most extensively studied observable in relativistic nucleusnucleus collisions and was measured in different experiments in the last three decades. However, the high-statistics differential measurements of $v_{2}$ as a function of centrality, $p_{T}$, rapidity for different particle species are available only at two beam energy domains: RHIC/LHC $\left(\sqrt{s_{\mathrm{NN}}}=30-5200 \mathrm{GeV}\right)$ and SIS $\left(\sqrt{s_{\mathrm{NN}}}=1-2 \mathrm{GeV}\right)$. At the moment for the beam energy range from $\sqrt{s_{\mathrm{NN}}}=2$ to $62.4 \mathrm{GeV}$ one can plot the excitation function for differential elliptic flow $v_{2}\left(p_{T}\right)$ only for protons from midcentral $\mathrm{Au}+\mathrm{Au}$ collisions. This is shown in the left panel of Fig. 4, where the $v_{2}\left(p_{T}\right)$ data from three different experiments: FOPI (SIS) [23], E895 (AGS) [33] and STAR (RHIC) [36] are presented. We emphasize, however, that the available data are not derived under the same experimental conditions. The differences include: a) different methods for flow measurements, b) different rapidity coverage and c) different centrality selection: FOPI (15-29\%), E895 (12-25\%) and STAR $(10-40 \%)$. According to the STAR BES-I data $v_{2}\left(p_{T}\right)$ for protons changes relatively little as a function of beam energy in the range $\sqrt{s_{\mathrm{NN}}}=11.5-62.4 \mathrm{GeV}$. According to the hybrid transport + viscous hydrodynamics approach such $v_{2}\left(p_{T}\right)$ behavior may result from the interplay of the hydrodynamic and hadronic transport phase [39]. The calculations show that the hydrodynamically produced $v_{2}$ does vanish at low collision energies $\sqrt{s_{\mathrm{NN}}}=5-7.7 \mathrm{GeV}$. However, the transport dynamics become more important at lower energies and are able to compensate for the reduction of hydrodynamically produced $v_{2}$ flow $[39,41]$.
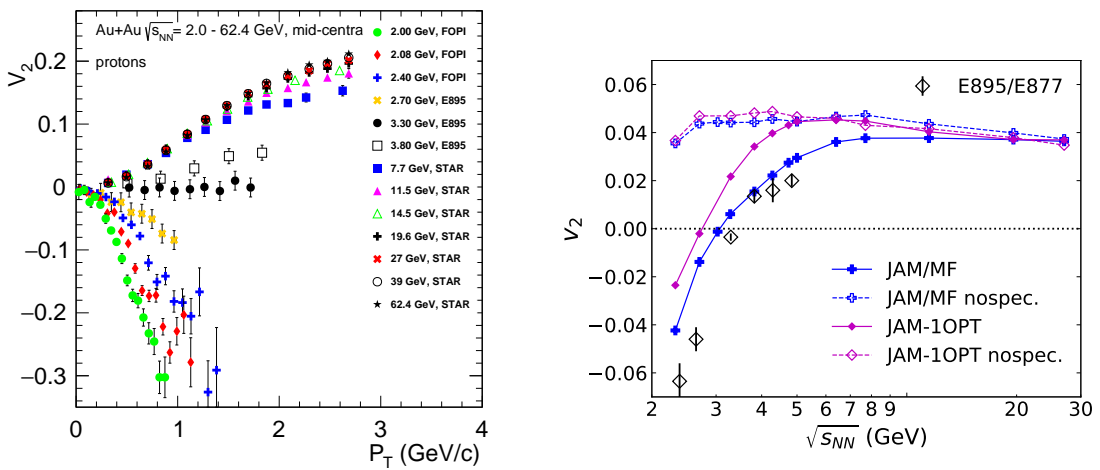

Figure 4. Left: Excitation function of differential elliptic flow $v_{2}\left(p_{T}\right)$ of protons from mid-central $\mathrm{Au}+\mathrm{Au}$ collisions at energies from $\sqrt{s_{\mathrm{NN}}}=2$ to $62.4 \mathrm{GeV}$. Data are taken from References: FOPI [23], E895 [33] and STAR [36]. Right: Excitation function of integral $v_{2}$ for nucleons in midcentral $\mathrm{Au}+\mathrm{Au}$ collisions $(4.6<\mathrm{b}<9.4 \mathrm{fm})$ from hadronic transport model JAM [30] with first-order phase transition and mean-field simulations with (closed symbols) and without spectator interactions (open symbols). Figure is taken from [30]

At lower beam energies $\sqrt{s_{\mathrm{NN}}}<11 \mathrm{GeV}$, shadowing effects by the spectator matter play an important role for the generation of elliptic flow. In the energy range $\sqrt{s_{\mathrm{NN}}}=11.5-2$ $\mathrm{GeV}$, the passage time $t_{\text {pass }}$ increases from $2 \mathrm{fm} / \mathrm{c}$ to $16 \mathrm{fm} / \mathrm{c}$. Right panel of Fig. 4 summarizes the effect of spectator shadowing on the elliptic flow of nucleons at midrapidity as a function of beam energy. The results were obtained using the hadronic transport model JAM [30] for mean-field simulation as well as simulations with a first-order phase transition. For a broad range of beam energies $\left(\sqrt{s_{\mathrm{NN}}}=2-7 \mathrm{GeV}\right)$, the elliptic flow results can be understood in terms of a delicate balance between (i) the ability of pressure developed early in the 
reaction zone, to effect a rapid transverse expansion of nuclear matter, and (ii) the passage time $t_{\text {pass }}$ for removal of the shadowing of participant hadrons by the projectile and target spectators $[29,30,33]$. The characteristic time for the development of expansion perpendicular to the reaction plane is $\sim R / c_{s}$, where the speed of sound $c_{s}=\sqrt{\partial P / \partial \varepsilon}, R$ is the nuclear radius, $P$ is the pressure and $\varepsilon$ is the energy density [29,33]. If the passage time $t_{\text {pass }}$ is long compared to the expansion time, spectator nucleons serve to block the path of participant hadrons emitted toward the reaction plane, and nuclear matter is squeezed-out perpendicular to this plane giving rise to negative elliptic flow $\left(v_{2}<0\right)$. The squeeze-out contribution should then reflect the ratio $c_{s} /\left(\gamma_{0} \beta_{0}\right)$. This is put into evidence in left panel of Fig. 5 where the differential elliptic flow values $v_{2}\left(p_{T}\right)$ for protons from FOPI experiment, shown for beam energies of $E_{\text {lab }}=0.6,0.8,1.0,1.2$ and $1.49 \mathrm{AGeV}$ in Fig. 4 , are plotted as a function of the normalized center-of-mass (c.m.) transverse momentum (per nucleon) $p_{T}^{0}=p_{T} / m_{p} \cdot\left(\gamma_{0} \beta_{0}\right)=$ $t_{\text {pass }} * p_{T} / m_{p}$. The rather good scaling observed suggest that $c_{s}$ does not change significantly over beam energy range $0.6-1.5 \mathrm{AGeV}$.
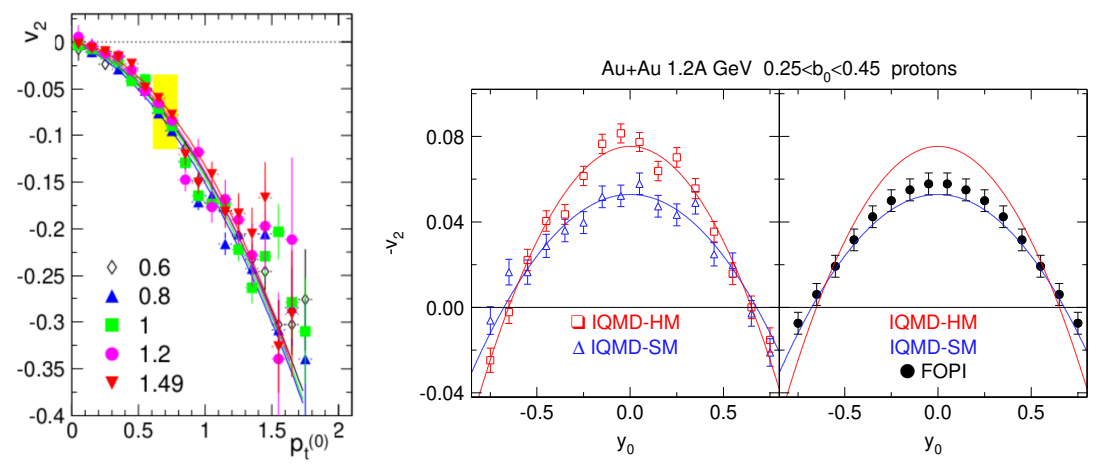

Figure 5. Left: Excitation function of differential elliptic flow $v_{2}\left(p_{T}^{0}\right)$ of protons from mid-central $\mathrm{Au}+\mathrm{Au}$ collisions $\left(E_{l a b}=0.6,0.8,1.2,1.45 \mathrm{AGeV}\right)$, where $p_{T}^{0}$ is the normalized center-of-mass (c.m.) transverse momentum (per nucleon). This is the same data points from FOPI experiment [23], plotted in the left panel of Fig. 4. The figure is taken from [23]. Right: IQMD model calculations (open symbols) for a hard (HM) and a soft (SM) EoS for elliptic flow signal $-v_{2}$ of protons as a function of the normalized rapidity $y_{0}$ for mid-central $\mathrm{Au}+\mathrm{Au}$ collisions at $E_{l a b}=1.2 \mathrm{AGeV}$. Lines show the fit results assuming a quadratic dependence $v_{2}\left(y_{0}\right)=v_{20}+v_{22} \Delta y_{0}^{2}$. The obtained fit results (lines) in comparison with the experimental data (closed symbols) measured with the FOPI experiment (GSI) [46]. The figures are taken from [47]

The elliptic flow at SIS energies $\left(0.4<E_{\text {lab }}<1.5 \mathrm{AGeV}\right.$ or $\left.\sqrt{s_{\mathrm{NN}}}=1-2.5 \mathrm{GeV}\right)$ is sensitive to the mean field and to the equation of state. Consequently, comparisons of results of high-statistics differential measurements of $v_{2}$ to model calculations can provide important constraints for the EOS. Such comparisons have been carried out for the beam energy range $0.4<E_{l a b}<1.49 \mathrm{AGeV}$ using the data from FOPI experiment at SIS [47]. The precision in interpreting the measured high-statistics differential $v_{2}$ data has been demonstrated by the FOPI Collaboration [46, 47]. In contrast to previous work [23], they used not only protons, but also two- and three-nucleon clusters that are emitted in $\mathrm{Au}+\mathrm{Au}$ collisions at $0.4<$ $E_{l a b}<1.49 \mathrm{AGeV}$ and that have larger flow signals than single nucleons [47]. They used not only the mid-rapidity data [23], but explored the strong dependence of $v_{2}$ on rapidity [46].

As an example the closed symbols on the right panel of Fig. 5 show the FOPI data for elliptic flow signal $-v_{2}$ of protons as a function of the normalized rapidity $y_{0}$ (rapidity normalized to the projectile rapidity in the c.m. system) for mid-central Au+Au collisions at $E_{\text {lab }}=1.2 \mathrm{AGeV}$. The data shows that elliptic flow $v_{2}$ as a function of rapidity $y_{0}$ can be well 


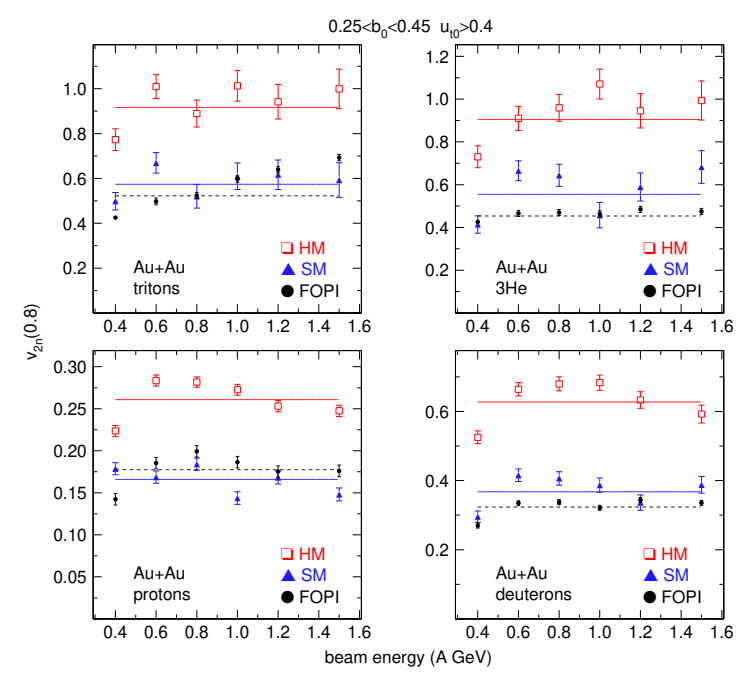

Figure 6. Elliptic flow parameter $v_{2 n}$ for protons, deuterons, tritons and ${ }^{3} \mathrm{He}$ as function of incident beam energy for FOPI experimental data and IQMD model calculations. The figure is taken from [47]

described by a quadratic fit $v_{2}=v_{20}+v_{22} \cdot y_{0}^{2}$. The open symbols show the results of Isospin Quantum Molecular Dynamics (IQMD) model calculations for a hard (HM) and a soft (SM) EoS [47]. They introduced the new quantity $v_{2 n}$ defined by $v_{2 n}=\left|v_{20}\right|+\left|v_{22}\right|$, which combines the information contained in the amplitude and the rapidity dependence of $v_{2}$. The value of $v_{2 n}$ was found to be very sensitive to the incompressibility $K_{0}$ and the in-medium nucleonnucleon cross section [47]. Fig. 6 presents the $v_{2 n}$ value for protons, deuterons, tritons and ${ }^{3} \mathrm{He}$ as function of incident beam energy for FOPI experimental data and IQMD model calculations. The dependence of $v_{2 n}$ on the incident energy in the interval 0.4 to $1.5 \mathrm{AGeV}$ covered by FOPI is fairly flat. This supports the previous conclusion based on the passage time scaling, that $c_{s}$ does not change significantly over beam energy range $0.6-1.5 \mathrm{AGeV}$. The discriminating power of new variable $v_{2 n}$ between the soft and stiff parametrizations of the symmetric-matter EoS looks very convincing [47].

\section{Triangular flow}

Similar to directed flow [22] one may expect to observe two components in triangular flow $v_{3}(y)=v_{3}^{\text {odd }}(y)+v_{3}^{\text {even }}(y)$. The rapidity-even component $v_{3}^{\text {even }}\left(v_{3}^{\text {even }}(y)=v_{3}^{\text {even }}(-y)\right)$ arises from event-by-event fluctuations in the initial nuclei and it is proportional to the fluctuationsdriven $\varepsilon_{3}$ of the system. According to hybrid transport + viscous hydrodynamics model calculations [39] the rapidity-even component $v_{3}^{\text {even }}$ is expected to be more sensitive to the viscous damping (than $v_{2}$ ) and might be an ideal observable to probe the formation of a QGP and the pressure gradients in the early plasma phase [39, 41]. The calculations show that the hydrodynamically produced $v_{3}^{e v e n}$ does vanish at low collision energies $\sqrt{s_{\mathrm{NN}}}=5-7.7 \mathrm{GeV}$ and there is no $v_{3}^{\text {even }}$ signal generated by the transport dynamics in the hadronic phase $[39,41]$.

These conclusions [39] are supported by the STAR measurements of $v_{3}^{2}\{2\}$ for charged hadrons based on BES-I RHIC data. Fig. 7 left shows the variation of $v_{3}^{2}\{2\}$ for charged hadrons produced at mid-rapidity from $7.7 \mathrm{GeV}$ up to $2.76 \mathrm{TeV}$ for different bins in collision centrality [38]. At low energies $\sqrt{s_{\mathrm{NN}}}<14.5 \mathrm{GeV}$, the $v_{3}^{2}\{2\}$ become consistent with zero 

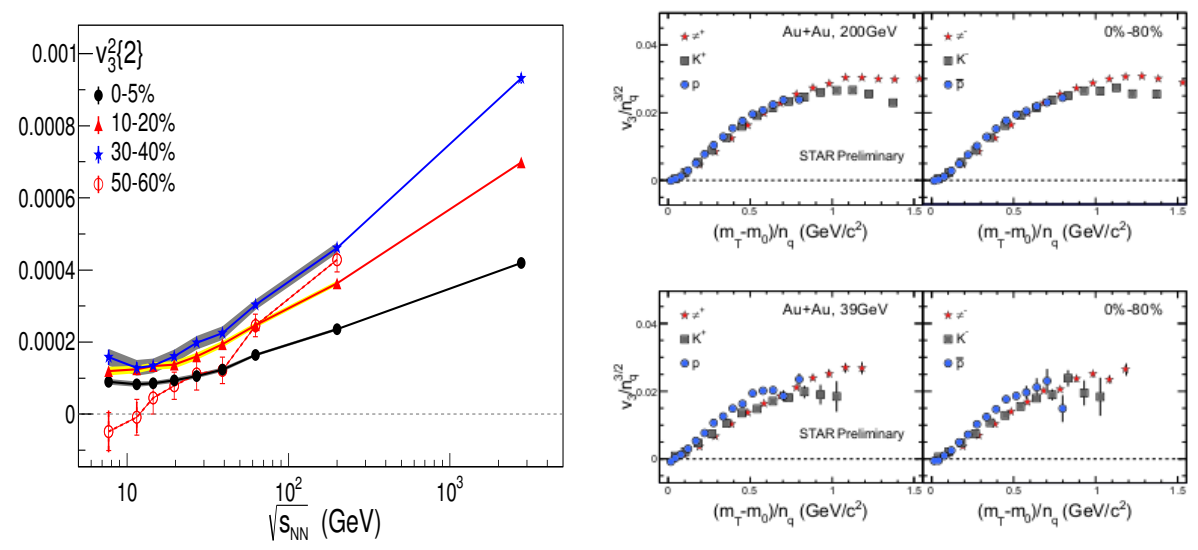

Figure 7. Left: The variation of $v_{3}^{2}\{2\}$ for charged hadrons produced at mid-rapidity from $7.7 \mathrm{GeV}$ up to $2.76 \mathrm{TeV}$ [34] for different bins in collision centrality. The figure is taken from [38] Right: Quark-number $\left(n_{q}\right)$ scaled triangular flow, $v_{3} / n_{q}^{3 / 2}$ versus $\left(m_{T}-m_{0}\right) / n_{q}$, for charged pions, kaons and (anti)protons emerged from $0-80 \%$ central $\mathrm{Au}+\mathrm{Au}$ collisions at $\sqrt{s_{\mathrm{NN}}}=200 \mathrm{GeV}$ (top panels) and at $\sqrt{s_{\mathrm{NN}}}=39 \mathrm{GeV}$ (bottom panels). The left panels represent the results for particles and right panels for anti-particles. The figure is taken from [40]

in 50-60\% peripheral collisions. This result is consistent with the idea of absence of a low viscosity QGP phase in low energy peripheral collisions [39]. For more central collisions, the values of $v_{3}^{2}\{2\}$ are positive and change little from $19.6 \mathrm{GeV}$ to $7.7 \mathrm{GeV}$. For the energies above $19.6 \mathrm{GeV}$, the values of $v_{3}^{2}\{2\}$ linearly increase with the $\log \left(\sqrt{s_{\mathrm{NN}}}\right)$ for all bins in collision centrality [38]. Fig. 7 right shows the preliminary data from STAR collaboration for $v_{3}$ of identified charged hadrons from $\mathrm{Au}+\mathrm{Au}$ collisions at $\sqrt{s_{\mathrm{NN}}}=200 \mathrm{GeV}$ (upper panels) and $39 \mathrm{GeV}$ (lower panels) [40]. The left panels represent the results for particles and right panels for anti-particles. For $200 \mathrm{GeV}$ the measured $v_{3}$ values follow the $v_{3} / n_{q}^{3 / 2}$ versus $\left(m_{T}-m_{0}\right) / n_{q}$ scaling. However, for $39 \mathrm{GeV}$ data one can see that the scaling is not so perfect.
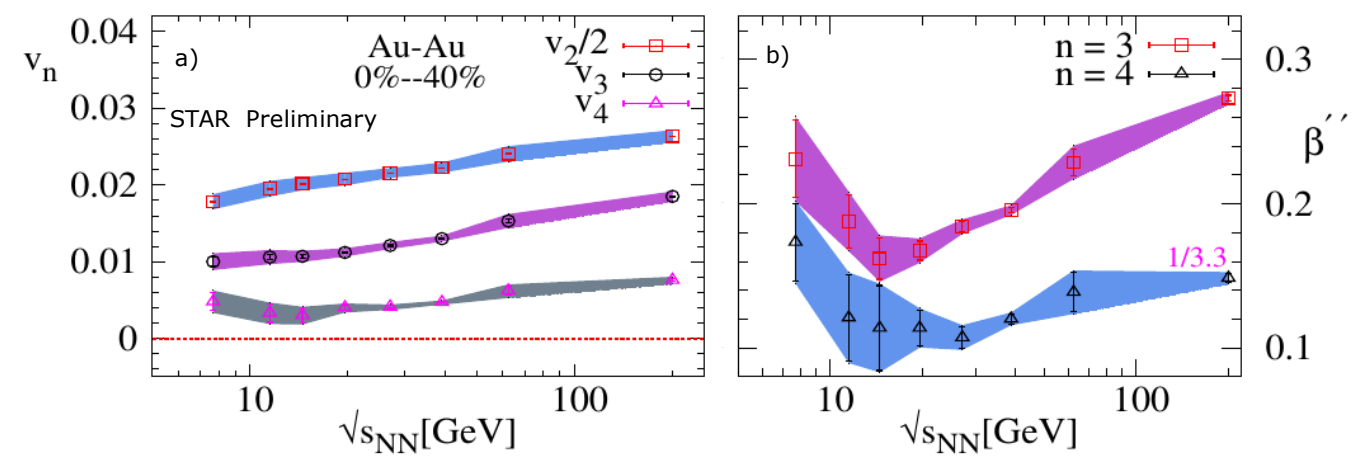

Figure 8. $\sqrt{s_{\mathrm{NN}}}$ dependence of the $p_{T}$-integrated $v_{n}$ (left panel) and the estimated viscous coefficient $\beta^{\prime \prime} \propto \eta / s$ (right panel). Results are shown for $0-40 \%$ central Au+Au collisions; the shaded lines are the systematic uncertainty. The figure is taken from [24] 
The left panel of Fig. 8 shows the preliminary STAR data for the $\sqrt{s_{\mathrm{NN}}}$ dependence of the $p_{T}$ integrated $v_{n}$ of charged hadrons from $0-40 \%$ central $\mathrm{Au}+\mathrm{Au}$ collisions [24]. It shows an essentially monotonic trend for $v_{2}, v_{3}$ and $v_{4}$ with $\sqrt{s_{\mathrm{NN}}}$ as might be expected for a temperature increase as $\sqrt{s_{\mathrm{NN}}}$ increases [24]. The same authors applied the "acoustic scaling" to $v_{n}$ data in order to estimate the $\sqrt{s_{\mathrm{NN}}}$ dependence of $\eta / s$. Using the observation that $\varepsilon_{n}$ changes very slowly with beam energy and the measurements for two different harmonics $n$ and $n^{\prime}\left(n \neq n^{\prime}\right)$ the Eq.1 can be simplified [24] to the viscous coefficient $\beta^{\prime \prime} \propto(\eta / s) \propto\left(d N_{c h} / d \eta\right)^{1 / 3} \ln \left(v_{n}^{1 / n} / v_{n^{\prime}}^{1 / n^{\prime}}\right)$. The right panel of Fig. 8 shows the $\sqrt{s_{\mathrm{NN}}}$ dependence of the viscous parameter $\beta$ " extracted from the $\ln \left(v_{3}^{1 / 3} / v_{2}^{1 / 2}\right)$ and $\ln \left(v_{4}^{1 / 4} / v_{2}^{1 / 2}\right)$ results from the left panel of Fig. 8. In contrast to $v_{n}$, the excitation function of the viscous parameter $\beta$ " shows a non-monotonic behavior over the same beam energy range. A similar non-monotonic trend for $\eta / s$ has been observed in the hybrid viscous hydrodynamical calculations [41], tuned to describe the STAR BES-I $v_{2}$ data.

At low beam energies available at SIS $\left(\sqrt{s_{\mathrm{NN}}}=1-2.5 \mathrm{GeV}\right)$ one clearly see the rapidity-odd component of $v_{3}^{\text {odd }}(y)\left(v_{3}^{\text {odd }}(y)=-v_{3}^{\text {odd }}(-y)\right)$. As an example Fig. 9 shows the results of HADES experiment at SIS for triangular flow $\left(v_{3}\right)$ of protons in semi-central $(20-30 \%)$ $\mathrm{Au}+\mathrm{Au}$ collisions at $E_{l a b}=1.23 \mathrm{AGeV}\left(\sqrt{s_{\mathrm{NN}}}=2.3 \mathrm{GeV}\right)$ [45]. The right panel of Fig. 9 shows the $v_{3}^{\text {odd }}$ of protons as a function of $p_{T}$ in the rapidity interval $-0.45<y_{c m}<-0.35$ together with UrQMD 3.4 model simulations with two parametrization of EoS [48]. The discriminating power of $v_{3}^{\text {odd }}$ between the soft and stiff parametrizations of the symmetric-matter EoS looks very convincing [48].
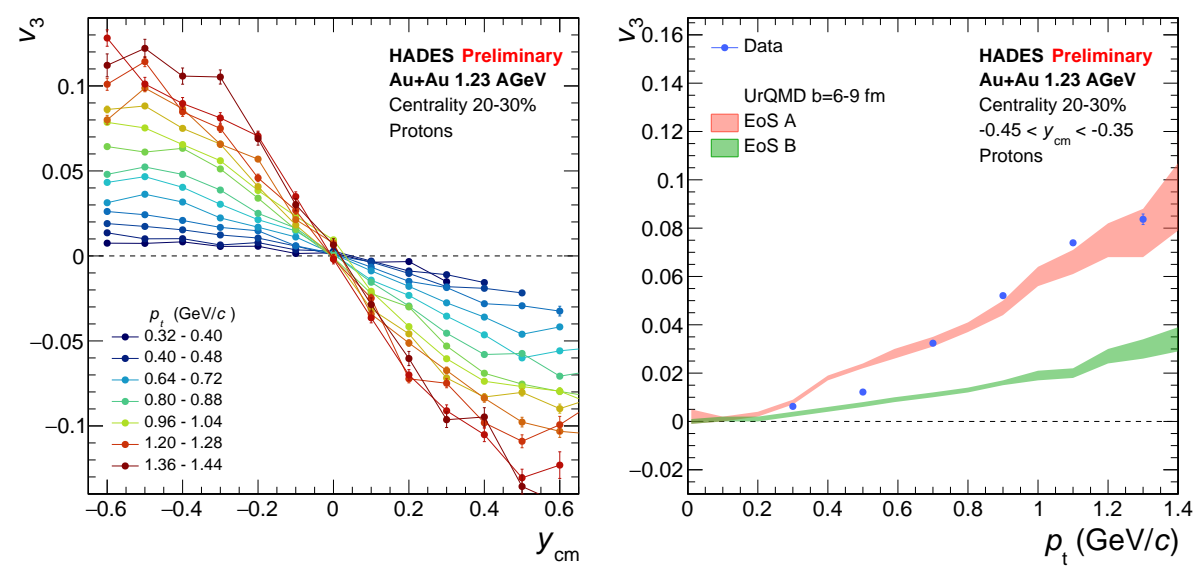

Figure 9. Left: HADES results for triangular flow $\left(v_{3}^{\text {odd }}\right)$ of protons in semi-central $(20-30 \%) \mathrm{Au}+\mathrm{Au}$ collisions at $1.23 \mathrm{AGeV}$ as a function of the centre-of-mass rapidity $y_{\mathrm{cm}}$ for different bins in transverse momentum as indicated. Right: $v_{3}^{\text {odd }}$ of protons as a function of $p_{T}$ in the rapidity interval $-0.45<$ $y_{c m}<-0.35$ together with UrQMD 3.4 model simulations with two parametrization of EoS [48]. The figures are taken from [45]

\section{Summary}

In this article, we briefly reviewed the recent results of the measurements of directed $\left(v_{1}\right)$, elliptic $\left(v_{2}\right)$ and triangular $\left(v_{3}\right)$ flow for collision energies from $\sqrt{s_{\mathrm{NN}}}=200 \mathrm{GeV}$ (RHIC) to $2 \mathrm{GeV}$ (SIS). The measured $v_{n}$ values are found to be sensitive to transport properties of the strongly interacting matter: the equation of state (EOS), the speed of sound $\left(c_{S}\right)$ and the 
value of $\eta / s$. However, in order the transport properties of the strongly interacting matter as a function of the temperature $T$ and baryon chemical potential $\mu_{B}$ further progress in the area of model calculations of $v_{n}$ is needed. On the experimental side, one need new highstatistics differential measurements of $v_{n}$ as a function of centrality, $p_{T}$, rapidity for different particle species in the high baryon density region $\sqrt{s_{\mathrm{NN}}}=2-11 \mathrm{GeV}$. The results of such measurements will be available soon from STAR experiment at RHIC (BES-II program), BM@N and MPD experiments at NICA [11] and CBM experiment at FAIR [12].

Acknowledgments. The reported study was funded by RFBR according to the research project No 18-02-00086 and the Ministry of Science and Higher Education of the Russian Federation, grant No 3.3380.2017/4.6.

\section{References}

[1] J. Adams et al. [STAR Collaboration], Nucl. Phys. A 757 (2005) 102

[2] K. Adcox et al. [PHENIX Collaboration], Nucl. Phys. A 757 (2005) 184

[3] C. Gale, S. Jeon, B. Schenke, P. Tribedy and R. Venugopalan, Phys. Rev. Lett. 110 (2013) no. 1,012302

[4] U. Heinz and R. Snellings, Ann. Rev. Nucl. Part. Sci. 63 (2013) 123

[5] Y. Aoki, G. Endrodi, Z. Fodor, S. D. Katz and K. K. Szabo, Nature 443 (2006) 675

[6] E. S. Bowman and J. I. Kapusta, Phys. Rev. C 79 (2009) 015202

[7] X. Luo, Nucl. Phys. A 956 (2016) 75

[8] D. Keane, J. Phys. Conf. Ser. 878 (2017) no.1, 012015.

[9] H. Caines, Nucl. Phys. A 967 (2017) 121.

[10] V. Klochkov et al. [NA61/SHINE Collaboration], Nucl. Phys. A 982 (2019) 439

[11] V. D. Kekelidze, Phys. Part. Nucl. 49 (2018) no.4, 457.

[12] T. Ablyazimov et al. [CBM Collaboration], Eur. Phys. J. A 53 (2017) no.3, 60

[13] S. Voloshin and Y. Zhang, Z. Phys. C 70 (1996) 665

[14] S. A. Voloshin, A. M. Poskanzer and R. Snellings, arXiv:0809.2949 [nucl-ex].

[15] R. Snellings, J. Phys. G 41 (2014) no.12, 124007

[16] R. Derradi de Souza, T. Koide and T. Kodama, Prog. Part. Nucl. Phys. 86 (2016) 35

[17] R. A. Lacey, A. Taranenko, J. Jia, D. Reynolds, N. N. Ajitanand, J. M. Alexander, Y. Gu and A. Mwai, Phys. Rev. Lett. 112 (2014) no.8, 082302

[18] R. A. Lacey, D. Reynolds, A. Taranenko, N. N. Ajitanand, J. M. Alexander, F. H. Liu, Y. Gu and A. Mwai, J. Phys. G 43 (2016) no.10, 10LT01

[19] J. Adam et al. [STAR Collaboration], Phys. Lett. B 784 (2018) 26

[20] J. Adam et al. [STAR Collaboration], arXiv:1901.08155 [nucl-ex].

[21] D. Teaney and L. Yan, Phys. Rev. C 83 (2011) 064904

[22] S. Singha, P. Shanmuganathan and D. Keane, Adv. High Energy Phys. 2016 (2016) 2836989

[23] A. Andronic et al. [FOPI Collaboration], Phys. Lett. B 612 (2005) 173

[24] N. Magdy [STAR Collaboration], J. Phys. Conf. Ser. 779 (2017) no.1, 012060.

[25] R. A. Lacey, P. Liu, N. Magdy, M. Csanád, B. Schweid, N. N. Ajitanand, J. Alexander and R. Pak, Universe 4, no. 1, 22 (2018)

[26] R. A. Lacey and A. Taranenko, PoS CFRNC 2006 (2006) 021

[27] A. Adare et al. [PHENIX Collaboration], Phys. Rev. C 93 (2016) no.5, 051902

[28] L. Adamczyk et al. [STAR Collaboration], Phys. Rev. Lett. 120 (2018) no.6, 062301

[29] R. A. Lacey, Nucl. Phys. A 774 (2006) 199 
[30] C. Zhang, J. Chen, X. Luo, F. Liu and Y. Nara, Phys. Rev. C 97 (2018) no.6, 064913

[31] D. H. Rischke, Nucl. Phys. A 610 (1996) 88C

[32] H. Stoecker, Nucl. Phys. A 750 (2005) 121

[33] C. Pinkenburg et al. [E895 Collaboration], Phys. Rev. Lett. 83 (1999) 1295

[34] L. Adamczyk et al. [STAR Collaboration], Phys. Rev. C 86 (2012) 054908

[35] L. Adamczyk et al. [STAR Collaboration], Phys. Rev. C 88 (2013) 014902

[36] L. Adamczyk et al. [STAR Collaboration], Phys. Rev. C 93 (2016) no.1, 014907 doi:10.1103/PhysRevC.93.014907

[37] P. Shanmuganathan [STAR Collaboration], Nucl. Phys. A 956, 260 (2016)

[38] L. Adamczyk et al. [STAR Collaboration], Phys. Rev. Lett. 116 (2016) no.11, 112302

[39] J. Auvinen and H. Petersen, Phys. Rev. C 88 (2013) no.6, 064908

[40] X. Sun [STAR Collaboration], J. Phys. Conf. Ser. 535 (2014) 012005.

[41] I. A. Karpenko, P. Huovinen, H. Petersen and M. Bleicher, Phys. Rev. C 91 (2015) no.6, 064901

[42] STAR Note 598. https://drupal.star.bnl.gov/STAR/starnotes/public/sn0598

[43] C. Yang [STAR Collaboration], Nucl. Phys. A 967 (2017) 800.

[44] K. Meehan [STAR Collaboration], Nucl. Phys. A 967 (2017) 808

[45] B. Kardan [HADES Collaboration], Nucl. Phys. A 982 (2019) 431

[46] W. Reisdorf et al. [FOPI Collaboration], Nucl. Phys. A 876, 1 (2012)

[47] A. Le Fèvre, Y. Leifels, W. Reisdorf, J. Aichelin and C. Hartnack, Nucl. Phys. A 945 (2016) 112

[48] P. Hillmann, J. Steinheimer and M. Bleicher, J. Phys. G 45 (2018) no.8, 085101 